ARTICLE

\title{
Induced superconductivity in high-mobility two-dimensional electron gas in gallium arsenide heterostructures
}

\author{
Zhong Wan ${ }^{1}$, Aleksandr Kazakov ${ }^{1}$, Michael J. Manfra ${ }^{1,2,3,4}$, Loren N. Pfeiffer ${ }^{5}$, Ken W. West ${ }^{5}$ \\ \& Leonid P. Rokhinson $1,2,4$
}

Search for Majorana fermions renewed interest in semiconductor-superconductor interfaces, while a quest for higher-order non-Abelian excitations demands formation of superconducting contacts to materials with fractionalized excitations, such as a two-dimensional electron gas in a fractional quantum Hall regime. Here we report induced superconductivity in high-mobility two-dimensional electron gas in gallium arsenide heterostructures and development of highly transparent semiconductor-superconductor ohmic contacts. Supercurrent with characteristic temperature dependence of a ballistic junction has been observed across $0.6 \mu \mathrm{m}$, a regime previously achieved only in point contacts but essential to the formation of well separated non-Abelian states. High critical fields (>16 T) in NbN contacts enables investigation of an interplay between superconductivity and strongly correlated states in a two-dimensional electron gas at high magnetic fields.

\footnotetext{
${ }^{1}$ Department of Physics and Astronomy, Purdue University, West Lafayette, Indiana 47907, USA. ${ }^{2}$ Department of Electrical Engineering, Purdue University, West Lafayette, Indiana 47907, USA. ${ }^{3}$ Department of Materials Engineering, Purdue University, West Lafayette, Indiana 47907, USA. ${ }^{4}$ Birck Nanotechnology Center, Purdue University, West Lafayette, Indiana 47907, USA. ${ }^{5}$ Department of Electrical Engineering, Princeton University, Princeton, New Jersey 08544, USA. Correspondence and requests for materials should be addressed to L.P.R. (email: leonid@purdue.edu)
} 
ntroduction of Josephson field effect transistor concept ${ }^{1}$ sparked active research on proximity effects in semiconductors. Induced superconductivity and electrostatic control of critical current has been demonstrated in twodimensional gases in InAs ${ }^{2,3}$, graphene ${ }^{4}$ and topological insulators $^{5-8}$, and in one-dimensional systems ${ }^{9-11}$ including quantum spin Hall edges ${ }^{12,13}$. Recently, interest in superconductor-semiconductor interfaces was renewed by the search for Majorana fermions ${ }^{14,15}$, which were predicted to reside at the interface ${ }^{16-18}$. More exotic non-Abelian excitations, such as parafermions (fractional Majorana fermions) ${ }^{19-21}$ or Fibonacci fermions may be formed when fractional quantum Hall edge states interface with superconductivity. Realization of a longsought regime of an interplay between superconductivity and strongly correlated states in a two-dimensional electron gas (2DEG) at high magnetic fields ${ }^{22-27}$ requires development of transparent superconducting contacts to high-mobility 2DEG, which remain superconducting at high magnetic fields.

Proximity effects in GaAs quantum wells have been intensively investigated in the past and Andreev reflection has been observed by several groups ${ }^{28-31}$. Unlike in InAs, where Fermi level $\left(E_{\mathrm{F}}\right)$ at the surface resides in the conduction band, in GaAs $E_{\mathrm{F}}$ is pinned in the middle of the gap, which results in a high Schottky barrier between a 2DEG and a superconductor and low transparency non-ohmic contacts. Heavy doping can move $E_{\mathrm{F}}$ into the conduction band and, indeed, superconductivity has been induced in heavily doped bulk $\mathrm{n}^{+}+\mathrm{GaAs}^{32}$. In quantum wells, similar results were obtained by annealing indium contacts ${ }^{33}$; however, the critical field of indium is $\sim 30 \mathrm{mT}$, well below the fields required to form quantum Hall effect (QHE) states.

In this article, we report the development of transparent superconducting ohmic contacts to high-mobility 2DEG in GaAs. The superconducting contact is type-II $\mathrm{NbN}$ with large critical field $>16 \mathrm{~T}$. Induced superconductivity is observed across $1.6 \mu \mathrm{m}$ of a $2 \mathrm{D}$ gas at zero field. From temperature dependence of the critical current and analysis of Andreev reflection, we estimate contact transparency parameter $Z \geqq 0.2$. Induced superconductivity is observed in magnetic fields up to $0.2 \mathrm{~T}$. At high magnetic fields, we observe deviations of longitudinal and Hall resistances from a similar data obtained with normal contacts, a clear indication of an interplay between superconductivity and QHE edge states.

\section{Results}

Heterostructures design. In conventional quantum well structures AlGaAs, barrier between 2DEG and the surface of the sample adds an extra $0.3 \mathrm{eV}$ to the Schottky barrier when contacts are defused from the top. We alleviated these problems by growing an inverted heterojunction structures, where a 2DEG resides at the GaAs/AlGaAs interface but the AlGaAs barrier with modulation doping is placed below the 2DEG, see Fig. 1b, where band diagram was calculated using a self-consistent Poisson solver ${ }^{34}$ (The program can be downloaded from http://www3.nd.edu/ gsnider/). Contacts are recessed into the top GaAs layer to bring the superconductor closer to the 2DEG. A thin layer of $\mathrm{AuGe}$ and $\mathrm{NbN}$ superconductor form low resistance ohmic contacts to the 2DEG after annealing. The inverted heterostructure increases the contact area of side contacts compared with quantum well structures by utilizing all GaAs layer above the heterointerface for carrier injection $(130 \mathrm{~nm}$ in our inverted heterostructure versus $20-30 \mathrm{~nm}$ in typical quantum wells, see Supplementary Fig. 1 and Supplementary Note 1).

Induced superconductivity. We report induced superconductivity in two devices from different wafers. Sample A has long $(70 \mu \mathrm{m})$ a

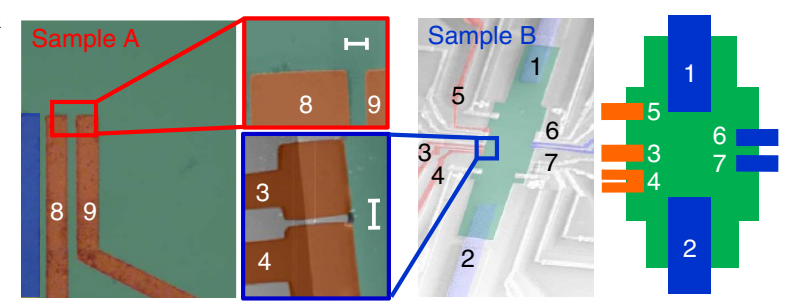

b

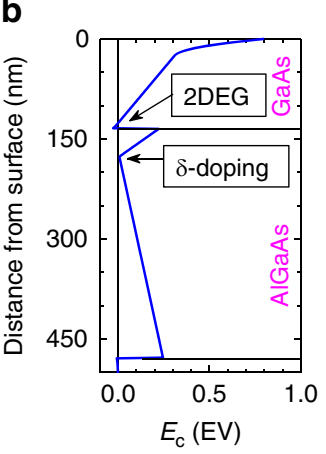

c

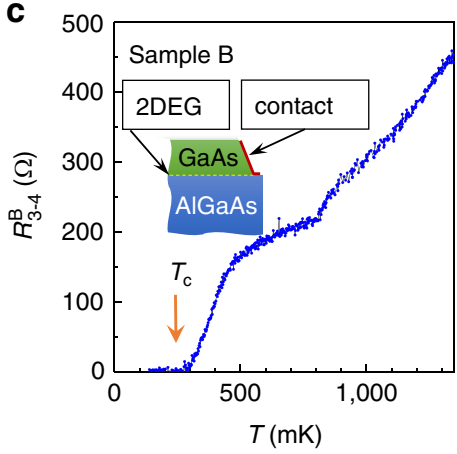

Figure 1 | Design and superconducting transition. (a) Scanning electron microscope images of test devices similar to samples A and B. Enlarged region for sample $B$ is an atomic force microscope image of a real sample. $2 \mathrm{D}$ gas regions are false-colour coded with green, superconducting and normal contacts are coded with orange and blue, respectively. Scale bar is $2 \mu \mathrm{m}$. (b) Simulation of the conduction band energy profile in the heterostructure. (c) T-dependence of resistance between contacts 3 and 4 in sample B measured with $10 \mathrm{nA}$ a.c. excitation. Superconducting transition is observed at $T_{\mathrm{c}} \approx 290 \mathrm{mK}$.

contacts separated by $1.6 \mu \mathrm{m}$ of $2 \mathrm{DEG}$, contacts to sample B are formed to the edge of a mesa with $0.6 \mu \mathrm{m}$ separation, see Fig. 1a. Details of device fabrication are described in Methods. When cooled down to $4 \mathrm{~K}$ in the dark, both the samples show resistance in excess of $1 \mathrm{M} \Omega$. After illumination with red light-emitting diode a $2 \mathrm{DEG}$ is formed and 2-terminal resistance drops to $<500 \Omega$. As shown in Fig. 1d, sample resistance $R B_{3-4}$ gradually decreases on cooldown from $4 \mathrm{~K}$ to the base temperature and the superconductor-2DEG-superconductor (S-2DEG-S) junction becomes superconducting at $T_{\mathrm{c}} \sim 0.3 \mathrm{~K}$.

Voltage-current $V(I)$ characteristics for two S-2DEG-S junctions (between contacts 8 and 9 for sample $A$, and 3 and 4 for sample B) are shown in Fig. 2. Both the samples show zero-resistance state at small currents with abrupt switching into resistive state at critical currents $I_{\mathrm{c}}=0.22$ and $0.23 \mu \mathrm{A}$ for samples A and $\mathrm{B}$, respectively. We attribute hysteresis in $V(I)$ characteristics to Joule heating in the normal state.

The most attractive property of a high-mobility 2DEG is large mean free path $l \gg \xi_{0}$, with $l=24 \mu \mathrm{m}$ and the Bardeen-CooperSchrieffer (BCS) coherence length $\xi_{0}=\hbar v_{\mathrm{F}} / \pi \Delta=0.72 \mu \mathrm{m}$ for sample B. Here $v_{\mathrm{F}}=\hbar \sqrt{2 \pi n} / m$ is the Fermi velocity, $n$ is a $2 \mathrm{D}$ gas density, $m$ is an effective mass and $\Delta=1.76 k_{\mathrm{B}} T_{\mathrm{c}}=46 \mu \mathrm{eV}$ is the induced superconducting gap. Evolution of $V(I)$ with $T$ is shown in Fig. 3a. Experimentally obtained $T$-dependence of $I_{c}$ is best described by the Kulik-Omelyanchuk theory for ballistic junctions $(L \ll l)$ (ref. 35), the blue curve in Fig. 3b. For comparison, we also plot $I_{\mathrm{c}}(T)$ dependence for the dirty limit $L \ll \sqrt{l \xi_{0}}$ (ref. 36), which exhibits characteristic saturation of $I_{\mathrm{c}}$ at low temperatures.

In short ballistic junctions, $L \ll \xi_{0} \ll l$ the product $I_{\mathrm{c}}(0) R N=$ $\pi \Delta / e$ does not depend on the junction length $L$. For $L \sim \xi_{0}$ this product is reduced by a factor $2 \xi_{0} /\left(L+2 \xi_{0}\right)$ (ref. 37). The 

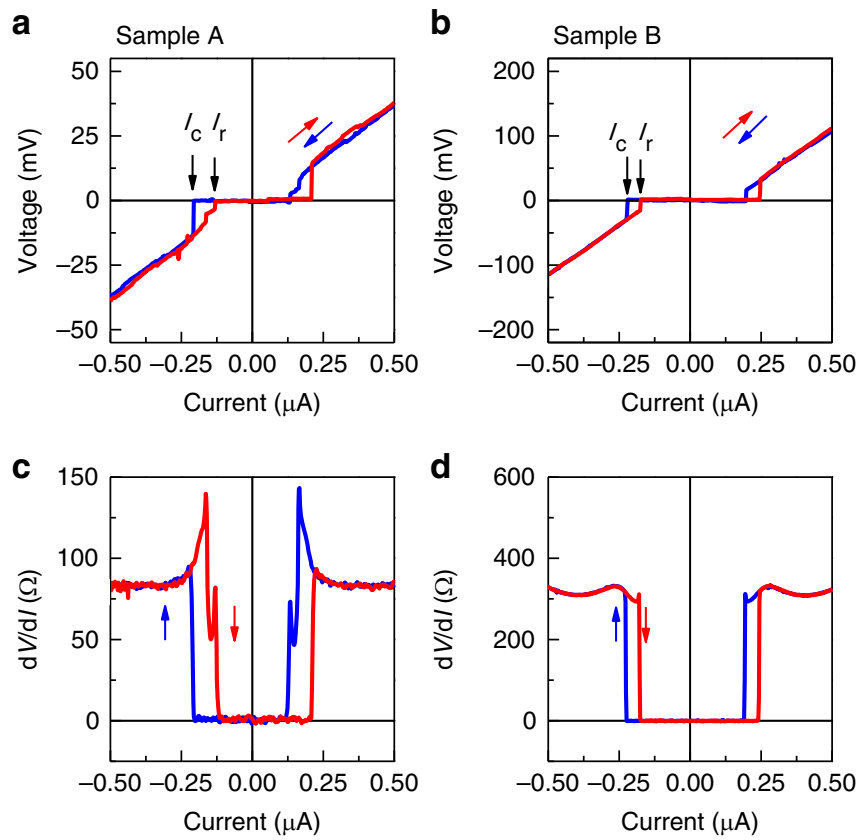

Figure 2 | Induced superconductivity in a high-mobility 2D electron gas

in GaAs. Voltage-current characteristics $(\mathbf{a}, \mathbf{b})$ and differential resistance $(\mathbf{c}, \mathbf{d})$ for samples $A(\mathbf{a}, \mathbf{c})$ and $B(\mathbf{b}, \mathbf{d})$. The conduction is measured between contacts (8-9) for sample $A$ and (3-4) for sample $B$. $d V / d /$ is measured with $I_{\text {a.c. }}=1 \mathrm{nA}$. Induced superconductivity with zero voltage is observed with critical currents $I_{\mathrm{c}} \sim 220 \mathrm{nA}$ for sample $\mathrm{A}$ and $I_{\mathrm{c}} \sim 230$ for sample B. Red (blue) traces are for current increasing (decreasing).

measured $I_{\mathrm{c}} R N=83 \mu \mathrm{V}$ for sample $\mathrm{B}$ is in a good agreement with an estimate $\pi \Delta / e \cdot 2 \xi_{0} /\left(L+2 \xi_{0}\right)=90 \mu \mathrm{V}$. For sample $\mathrm{A}$, the $I_{\mathrm{c}} R N=19 \mu \mathrm{V}$ while the estimated product is $\approx 50 \mu \mathrm{V}$. The reduction is consistent with the geometry of sample $\mathrm{A}$, where a region of the $2 \mathrm{DEG}$ with induced superconductivity is shunted by a large region of a 2 DEG in a normal state.

Transparency of a superconductor/2DEG interface. In onedimensional junctions, the induced gap $\Delta=\Delta_{0} \frac{\Gamma}{\Gamma+\Delta_{0}}$ depends on the broadening of Andreev levels within the semiconductor ${ }^{38}$ $\Gamma=\frac{\hbar \nu_{\mathrm{F}}}{L_{\mathrm{F} f}} D_{1} D_{2}$, where we introduce contacts transparencies $D_{1}$ and $D_{2}$. We assume for simplicity that $D_{1}=D_{2}=1 /\left(1+Z^{2}\right)$, where $0<Z<\infty$ is a interface barrier strength introduced in ref. 39, and Bagwell's effective channel length $L_{\text {eff }}=L+2 \xi_{0}$. Using $\mathrm{NbN}$ superconducting gap, $\Delta_{0}=2.02 k_{\mathrm{B}} T_{\mathrm{c}}^{0}(\mathrm{NbN}$ is a strong-coupling superconductor, $T_{\mathrm{c}}^{0}=11 \mathrm{~K}$ ) and $T_{\mathrm{c}}=0.3 \mathrm{~K}$ for $R_{3-4}^{\mathrm{B}}$ we obtain $Z=0.2$. This value is consistent with the fit of the $I_{\mathrm{c}}$ versus $T$-dependence with $D$ as a free parameter (Supplementary Fig. 2; Supplementary Note 2). Similar values of $Z$ can be estimated from the analysis of the shape of $\mathrm{d} I / \mathrm{d} V(V)$ characteristics at elevated temperatures, as shown in Fig. 3. At $T<T_{\mathrm{c}}^{0}$, Andreev reflection at S-2DEG interfaces results in an excess current flowing through the junction for voltage biases within the superconducting gap $\Delta_{0} / e$ and corresponding reduction of a differential resistance $\mathrm{d} V / \mathrm{d} I$ by a factor of 2 . In the presence of a tunnelling barrier, normal reflection competes with Andreev reflection and reduced excess current near zero bias, resulting in a peak in differential resistance. Within the BlonderTinkham-Klapwijk theory ${ }^{39}$, a flat $\mathrm{d} V / \mathrm{d} I(V)$ within $\Delta_{0} / e$, observed in our experiments, is expected only for contacts with very high transparency $Z<0.2$. For larger $Z>0.2$, a peak at low biases is expected (Supplementary Fig. 3, Supplementary Note 3). Several features of the experimental $I(V)$ need to be mentioned.

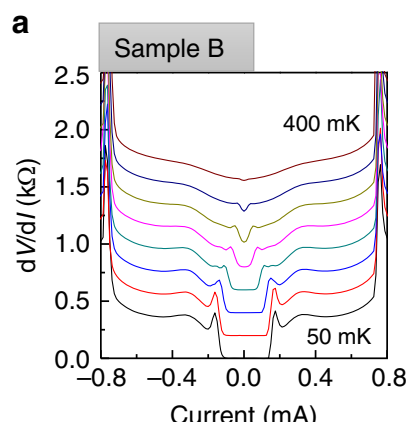

b
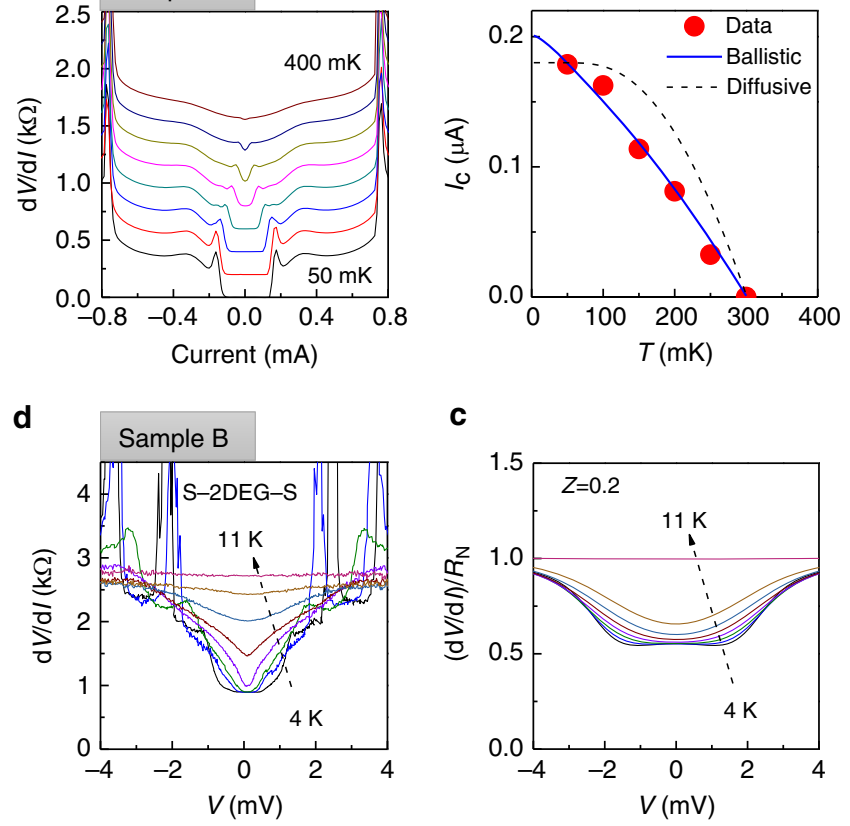

C

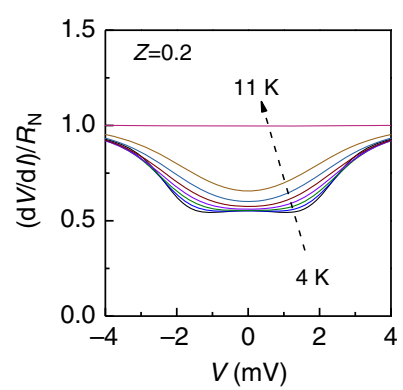

Figure 3 | Temperature dependence of superconductivity in a ballistic junction. (a) Evolution of the induced superconductivity with $T$ for sample B. The $R(I)$ curves are offset proportional to $T$ for $T>50 \mathrm{mK}$. (b) Temperature dependence of critical current $I_{\mathrm{c}}(T)$ is extracted from (a) and compared with the expected $T$-dependence for ballistic and diffusive regimes (reduced $I_{c}$ compared with Fig. 2 is due to larger $I_{\text {a.c. }}=10 \mathrm{nA}$ used in this experiment). (c) High-temperature data shows Andreev reflection (excess current and reduced $\mathrm{dV} / \mathrm{d} /$ around $V=0$. The curves are not offset. In $\mathbf{d}$, excess current is modelled within the Blonder-Tinkham-Klapwijk theory ${ }^{39}$ with $Z=0.2$.

First, we observe several sharp peaks in the resistance at high biases (around 2 and $4 \mathrm{mV}$ for $T=4 \mathrm{~K}$ ). Similar sharp resonances has been observed previously ${ }^{40}$, where authors attributed their appearance to the formation of Fabry-Pérot resonances between superconducting contacts. In our devices, the superconducting region is shunted by a low resistance $(<100 \Omega) 2 D E G$, thus appearance of $>10 \mathrm{k} \Omega$ resonances cannot be explained by resonant electron trapping between contacts. These resonances are also observed in $I(V)$ characteristics of a single S-2DEG interface (measured in the S-2DEG-N configuration between contacts 3 and 6, see Supplementary Fig. 3). Differential resistance does not change substantially across resonances, ruling out transport through a localized state. We speculate that in the contacts where these resonances are observed superconductivity is carried out by quasi-one-dimensional channels, and jumps in $I / V$ characteristics are due to flux trapping at high currents. This scenario is consistent with the observation that peaks shift to lower currents at higher fields, see Fig. 4. The second notable feature of our data is reduction of the zero-bias resistance by $\approx 2.6$ times at low temperatures, while Andreev reflection limits the reduction to the factor of 2 . We attribute this reduction to the multiple Andreev reflection between two closely spaced contacts, for contacts with larger separation $(20 \mu \mathrm{m})$ multiple Andreev reflection is suppressed and the reduction of resistance by a factor of 2 is observed (Supplementary Fig. 3).

Induced superconductivity in low magnetic fields. Finally, we present magnetic field dependence of induced superconductivity. The low-field data is shown in Fig. 4a,b, where black regions 
a

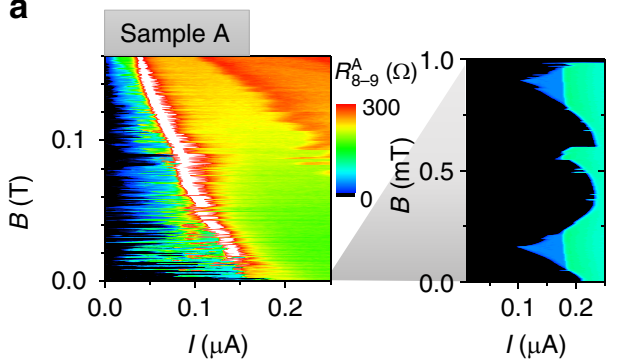

b

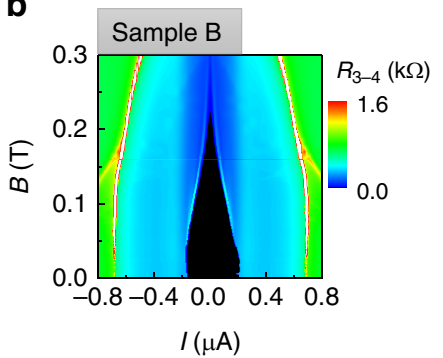

C

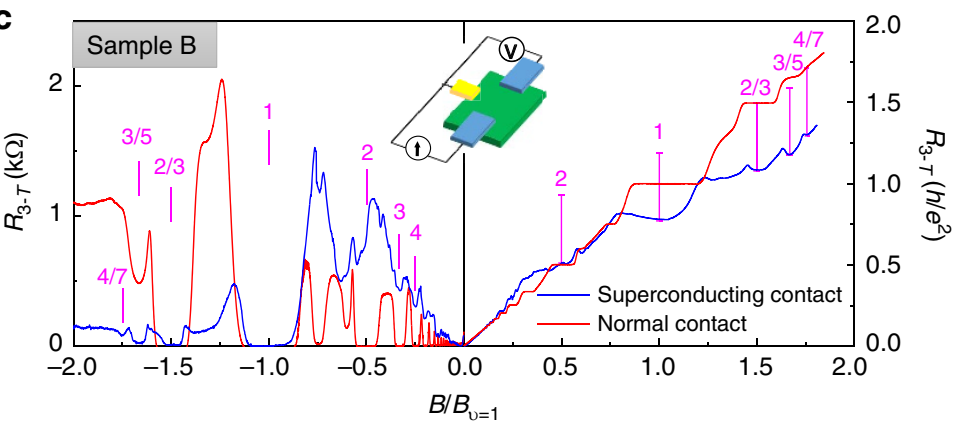

Figure 4 | Magnetic field dependence of induced superconductivity. (a,b) Differential resistance is measured as a function of $B$ and $I_{\text {d.c. }}$ for two samples at $40 \mathrm{mK}$. Induced superconductivity (black region) is observed up to $0.2 \mathrm{~T}$ in both the samples. (c) 3-terminal resistance for a sample with all normal contacts (red) and between normal and superconducting contacts in sample B (I (2-4) and V (4-1) in Fig. 1) is measured at 70 and 40 mK, respectively. $B<0(B>0)$ induces clockwise (counterclockwise) chiral edge channels, note resistance scales difference for two field directions.

correspond to zero-differential resistance. Induced superconductivity is suppressed at $\approx 0.2 \mathrm{~T}$ in both the samples. In sample $A$, a narrow region of a 2 DEG with induced superconductivity is confined between large $\mathrm{NbN}$ superconducting leads with rigid phases. Perpendicular magnetic field twists the phase in the 2DEG resulting in Fraunhofer-like oscillations of the critical current. In this sample, although the 2DEG extends beyond the narrow region between the contacts and $I_{c}$ does not decrease to zero and abrupt jumps in $I_{\mathrm{c}}$ reflect multiple flux jumps. The period of oscillations is $\sim 0.5 \mathrm{mT}$, which corresponds to an area of $4.1 \mu \mathrm{m}^{2}$, much smaller than the area of the $2 \mathrm{DEG}$ between the contacts $\left(\approx 120 \mu \mathrm{m}^{2}\right)$. This observation is consistent with the reduced $I_{\mathrm{c}} R N$ product measured for this sample as discussed above. In sample $B$, contacts are fabricated along the edge of the mesa and 2D gas is not enclosed between the contacts. Consequently, $I_{\mathrm{c}}$ is a smooth function of $B$.

Superconductivity and quantum Hall effect. Competition between superconductivity and chiral quantum Hall edge states is shown in Fig. 4c, where resistance is measured in a 3-terminal configuration over a wide range of magnetic fields. Simple Landauer-Büttiker model of edge states predicts zero resistance for negative and quantized Hall resistance for positive field direction for integer QHE and fractional QHE states, which is clearly seen in a sample with non-superconducting (AuGe) ohmic contacts (red curve). When a superconducting contact serves as a current injector (blue curve), integer $v=1$ and fractional $v=2 / 3$ and $3 / 5$ states are well developed for $B<0$, while the same states are not quantized at proper QHE values for $B>0$. If we assume that current injection via a superconducting contact results in an extra voltage offset at the contact by $V_{\text {off }} \approx \Delta_{\text {ind }} / e$, the measured voltage will be reduced by $V_{\text {off }}$. The magenta bars for $B>0$ indicate corrected resistance $\left(V-V_{\text {off }}\right) / I$ for $V_{\text {off }}=140 \mu \mathrm{V}$. While this offset may explain the measured values for fractional states, a twice smaller $V_{\text {off }}$ is needed to reconcile the resistance at $v=1$. Note that $\mathrm{NbN}$ critical field $B_{\mathrm{c}}>16 \mathrm{~T}$. At low fields, states $v=3,4$ and 5 have resistance minima for $B<0$, indicating a partial equilibration of chiral edge currents with the superconducting contact, while resistance near $v=2$ has a maximum. Zero resistance at $v=1$ and large resistance at $v=2$ are in contrast to the theoretical prediction that $v=2$ state should be stronger coupled with a superconducting contact than $v=1$ (ref. 23).

\section{Methods}

GaAs wafers design and parameters. The GaAs/AlGaAs inverted heterojunctions were grown by molecular beam epitaxy on semi-insulating (100) GaAs substrates with the heterointerface placed $130 \mathrm{~nm}$ below the surface and $\delta$-doping layer $30-40 \mathrm{~nm}$ below the GaAs/AlGaAs interface. Samples were fabricated from two wafers with density and mobility $n=2.7 \times 10^{11} \mathrm{~cm}^{-2}, \mu=2 \times 10^{6} \mathrm{~V} \mathrm{~s} \mathrm{~cm}^{-2}$ (sample A) and $n=1.7 \times 10^{11} \mathrm{~cm}^{-2}, \mu=4 \times 10^{6} \mathrm{~V} \mathrm{~s} \mathrm{~cm}^{-2}$ (sample B).

Fabrications of superconducting contacts. Superconducting contacts were defined by standard electron beam lithography. First, a $120 \mathrm{~nm}$-deep trench was created by wet etching. Next, samples were dipped into $\mathrm{HCl}: \mathrm{H}_{2} \mathrm{O}(1: 6)$ solution for $2 \mathrm{~s}$ and loaded into a thermal evaporation chamber, where Ti/AuGe $(5 / 50 \mathrm{~nm})$ was deposited. Finally, $70 \mathrm{~nm}$ of $\mathrm{NbN}$ was deposited by DC magnetron sputtering in $\mathrm{Ar} / \mathrm{N}_{2}(85 / 15 \%)$ plasma at a total pressure of $2 \mathrm{mTorr}$. Deposition conditions were optimized for producing high quality $\mathrm{NbN}$ films with $T_{\mathrm{c}}=11 \mathrm{~K}$ and $B_{\mathrm{c}}>15 \mathrm{~T}$, see Supplementary Fig. 4, and with minimal strain ${ }^{41}$. After metallization, contacts were annealed at $500^{\circ} \mathrm{C}$ for $10 \mathrm{~min}$ in a forming gas $\left(10 \% \mathrm{H}_{2}\right.$ in $\left.\mathrm{Ar}\right)$. Measurements were performed in a dilution refrigerator with the base temperature $<30 \mathrm{mK}$, high-temperature data was obtained in a variable temperature ${ }^{3} \mathrm{He}$ system. Samples were illuminated with red light-emitting diode at $4 \mathrm{~K}$ to form a $2 \mathrm{D}$ gas, 2 -terminal resistance drops from $>1 \mathrm{M} \Omega$ before illumination to $<500 \Omega$ after illumination.

\section{References}

1. Clark, T. D., Prance, R. J. \& Grassie, A. D. C. Feasibility of hybrid Josephson field effect transistors. J. Appl. Phys. 51, 2736-2743 (1980).

2. Takayanagi, H. \& Kawakami, T. Superconducting proximity effect in the native inversion layer on InAs. Phys. Rev. Lett. 54, 2449-2452 (1985).

3. Akazaki, T., Takayanagi, H., Nitta, J. \& Enoki, T. A Josephson field effect transistor using an InAs inserted channel $\operatorname{In}_{0.52} \mathrm{Al}_{0.48} \mathrm{As} / \mathrm{In}_{0.53} \mathrm{Ga}_{0.47}$ As inverted modulation-doped structure. Appl. Phys. Lett. 68, 418-420 (1996).

4. Heersche, H. B., Jarillo-Herrero, P., Oostinga, J. B., Vandersypen, L. M. K. \& Morpurgo, A. F. Bipolar supercurrent in graphene. Nature 446, 56-59 (2007).

5. Sacepe, B. et al. Morpurgo. Gate-tuned normal and superconducting transport at the surface of a topological insulator. Nat. Commun. 2, 575 (2011). 
6. Williams, J. R. et al. Unconventional Josephson effect in hybrid superconductor-topological insulator devices. Phys. Rev. Lett. 109, 056803 (2012).

7. Veldhorst, M. et al. Josephson supercurrent through a topological insulator surface state. Nat. Mater. 11, 1-4 (2012).

8. Qu, F. et al. Strong superconducting proximity effect in $\mathrm{Pb}-\mathrm{Bi}_{2} \mathrm{Te}_{3}$ hybrid structures. Sci. Rep 2, 339 (2012).

9. Doh, Y.-J. et al. Tunable supercurrent through semiconductor nanowires. Science 309, 272-275 (2005).

10. Jarillo-Herrero, P., van Dam, J. A. \& Kouwenhoven, L. P. Quantum supercurrent transistors in carbon nanotubes. Nature 439, 953-956 (2006)

11. Xiang, J., Vidan, A., Tinkham, M., Westervelt, R. M. \& Lieber., C. M. Ge/Si nanowire mesoscopic Josephson junctions. Nat. Nanotechnol. 1, 208-213 (2006).

12. Hart, S. et al. Induced superconductivity in the quantum spin hall edge. Nat. Phys. 10, 638-643 (2014).

13. Yu, W. et al. Superconducting proximity effect in inverted InAs/GaSb quantum well structures with Ta electrodes. preprint at arXiv:1402.7282 (2014).

14. Rokhinson, L. P., Liu, X. \& Furdyna., J. K. The fractional a.c. Josephson effect in a semiconductor-superconductor nanowire as a signature of Majorana particles. Nat. Phys. 8, 795-799 (2012).

15. Mourik, V. et al. Signatures of Majorana fermions in hybrid superconductorsemiconductor nanowire devices. Science 336, 1003-1007 (2012).

16. Fu, L. \& Kane, C. L. Josephson current and noise at a superconductor/ quantum-spin-Hall-insulator/superconductor junction. Phys. Rev. B 79, 161408 (2009).

17. Lutchyn, R. M., Sau, J. D. \& Sarma, S. D. Majorana fermions and a topological phase transition in semiconductor-superconductor heterostructures. Phys. Rev. Lett. 105, 077001 (2010).

18. Alicea, J. Majorana fermions in a tunable semiconductor device. Phys. Rev. B 81, 125318 (2010).

19. Clarke, D. J., Alicea, J. \& Shtengel, K. Exotic non-Abelian anyons from conventional fractional quantum Hall states. Nat. Commun. 4, 1348 (2012).

20. Mong, R. S. K. et al. Universal topological quantum computation from a superconductor-Abelian quantum hall heterostructure. Phys. Rev. X 4, 011036 (2014).

21. Zuo, Z.-W., Sheng, L. \& Xing., D. Y. Crossed Andreev reflections in superconductor and fractional quantum Hall liquids hybrid system. Solid State Commun. 184, 17-20 (2014).

22. Zyuzin, A. Y. Superconductor-normal-metal-superconductor junction in a strong magnetic field. Phys. Rev. B 50, 323-329 (1994).

23. Fisher., M. P. A. Cooper-pair tunneling into a quantum Hall fluid. Phys. Rev. B 49, 14550-14553 (1994).

24. Hoppe, H., Zülicke, U. \& Schön, G. Andreev reflection in strong magnetic fields. Phys. Rev. Lett. 84, 1804-1807 (2000).

25. Kim, E.-A., Vishveshwara, S. \& Fradkin., E. Cooper-pair tunneling in junctions of singlet quantum Hall states and superconductors. Phys. Rev. Lett. 93, 266803 (2004).

26. Giazotto, F., Governale, M., Zülicke, U. \& Beltram, F. Andreev reflection and cyclotron motion at superconductor/normal-metal interfaces. Phys. Rev. B 72, 054518 (2005).

27. van Ostaay, J. A. M., Akhmerov, A. R. \& Beenakker, C. W. J. Spin-triplet supercurrent carried by quantum Hall edge states through a Josephson junction. Phys. Rev. B 83, 195441 (2011).

28. Lenssen, K-M. H. et al. Andreev reflection at superconducting contacts to GaAs/AlGaAs heterostructures. Appl. Phys. Lett. 63, 2079-2081 (1993).

29. Moore, T. D. \& Williams, D. A. Andreev reflection at high magnetic fields. Phys. Rev. B 59, 7308-7311 (1999).

30. Verevkin, A. A. et al. Multiple Andreev reflection in hybrid AlGaAs GaAs structures with superconducting NbN contacts. Semiconductors 33, 551-554 (1999).

31. Takayanagi, H., Akazaki, T., Kawamura, M., Harada, Y. \& Nitta, J. Superconducting junctions using AlGaAs/GaAs heterostructures with high $\mathrm{h}_{c 2}$ NbN electrodes. Physica E 4, 922-926 (2002).

32. Kutchinsky, J., Taboryski, R., Srensen, C. B., Hansen, J. B. \& Lindelof, P. E. Experimental investigation of supercurrent enhancement in s-n-s junctions by non-equilibrium injection into supercurrent-carrying bound Andreev states. Physica C 352, 4-10 (2001).

33. Marsh, A. M., Williams, D. A. \& Ahmed, H. Supercurrent transport through a high-mobility two-dimensional electron gas. Phys. Rev. B 50, 8118-8121 (1994).

34. Tan, I.-H., Snider, G. L., Chang, L. D. \& Hu., E. L. A self-consistent solution of schrodinger-poisson equations using a nonuniform mesh. J. Appl. Phys. 68, 4071 (1990).

35. Kulik, I. O. \& Omel'anchuk, A. N. Properties of superconducting microbridges in the pure limit. Sov. J. Low Temp. Phys. 3, 459-461 (1977).

36. Kulik, I. O. \& Omel'yanchuk, A. N. Contribution to the microscopic theory of the Josephson effect in superconducting bridges. Sov. Phys. JETP 21, 96-97 (1975).

37. Bagwell, P. F. Suppression of the Josephson current through a narrow, mesoscopic, semiconductor channel by a single impurity. Phys. Rev. B 46, 12573-12586 (1992).

38. Sau, J. D., Tewari, S. \& Sarma, S. D. Experimental and materials considerations for the topological superconducting state in electron and hole doped semiconductors: searching for non-Abelian Majorana modes in 1d nanowires and 2d heterostructures. Phys. Rev. B 85, 064512 (2012).

39. Blonder, G. E., Tinkham, M. \& Klapwijk, T. M. Transition from metallic to tunneling regimes in superconducting microconstrictions: excess current, charge imbalance, and supercurrent conversion. Phys. Rev. B 25, 4515-4532 (1982).

40. Gao, J. R. et al. Superconductors coupled with a two-dimensional electron gas in GaAs/AlGaAs and InAs/AlGaSb heterostructures. Surf. Sci. 305, 470-475 (1994).

41. Glowacka, D. M. et al. Development of a NbN deposition process for superconducting quantum sensors. preprint at arXiv:1401.2292 (2014).

\section{Acknowledgements}

The work at Purdue was supported by the National Science Foundation grant DMR 1307247 (Z.W. and L.P.R.), by the Purdue Center for Topological Materials (Z.W.) and by the US Department of Energy, Office of Basic Energy Sciences, Division of Materials Sciences and Engineering under Awards DE-SC0008630 (A.K.) and DE-SC0006671 (M.J.M.). The work at Princeton was funded by the Gordon and Betty Moore Foundation through Grant GBMF 4420 and by the National Science Foundation MRSEC at the Princeton Center for Complex Materials.

\section{Author contributions}

L.P.R. and M.J.M conceived the experiments, Z.W. fabricated samples, Z.W. and L.P.R performed the experiments, Z.W. and L.P.R wrote the manuscript with comments from M.J.M, L.N.P. and K.W.W. designed and grew wafers, and A.K. contributed to the fabrication and low temperature experiments.

\section{Additional information}

Supplementary Information accompanies this paper at http://www.nature.com/ naturecommunications

Competing financial interests: The authors declare no competing financial interests.

Reprints and permission information is available online at http://npg.nature.com/ reprintsandpermissions/

How to cite this article: Wan, Z. et al. Induced superconductivity in high-mobility two-dimensional electron gas in gallium arsenide heterostructures. Nat. Commun. 6:7426 doi: $10.1038 /$ ncomms8426 (2015).

This work is licensed under a Creative Commons Attribution 4.0 International License. The images or other third party material in this article are included in the article's Creative Commons license, unless indicated otherwise in the credit line; if the material is not included under the Creative Commons license, users will need to obtain permission from the license holder to reproduce the material. To view a copy of this license, visit http://creativecommons.org/licenses/by/4.0/ 